\title{
Generalization of the Force Approach to Radiation Reaction
}

\author{
Gustavo V. López \\ Departamento de Física, Universidad de Guadalajara, Guadalajara, Mexico \\ Email: gulopez@udgserv.cencar.udg.mx
}

How to cite this paper: López, G.V. (2017) Generalization of the Force Approach to Radiation Reaction. Journal of Applied Mathematics and Physics, 5, 569-573. https://doi.org/10.4236/jamp.2017.53048

Received: January 22, 2017

Accepted: March 3, 2017

Published: March 6, 2017

Copyright $\odot 2017$ by author and Scientific Research Publishing Inc. This work is licensed under the Creative Commons Attribution International License (CC BY 4.0).

http://creativecommons.org/licenses/by/4.0/

\begin{abstract}
A new approach to radiation reaction for the correction of the linear and circular motion of a charged particle takes into account the emission of electromagnetic radiation due to its acceleration. This new formulation was based on expressing the radiation reaction force in terms of the external force rather than the acceleration of the charge. In this paper, a generalization of the radiation reaction force in terms of the external force approach is formulated for any arbitrary motion of the charged particle. This generalization includes the linear and circular acceleration cases previously investigated.
\end{abstract}

\section{Keywords}

Synchrotron Radiation, Radiation Reaction Force, Relativistic Charged Particle

\section{Introduction}

There is an old standing problem of radiation reaction [1] [2] [3] [4] consisting mainly in the that the usual approach, based on expressing the radiation reaction force in terms of the third time differentiation of the position of the charged particle, brings about of what is called a pre-acceleration of the charged particle even if there is not external force at all. However, experimentally one sees that as soon the external force is zero, the acceleration of the charged particle is zero too, and emission of electromagnetic radiation stops. This experimental fact led to propose an alternative approach [5] to the radiation reaction force. This new approach is based on expressing the radiation reaction force in terms of the external force acting on the charged particle, responsible for its acceleration. Although, this approach seems to point in the right direction, experimental verification of this theoretical idea is required. In reference [5], the approach was implemented for linear and circular acceleration of a charged particle. In this paper, a generalization of the idea is carried out, using the same method pre- 
sented in that paper, and the generalization takes the same form as Equatioin (1), but with the additional radiation reaction force term.

\section{Acceleration and Force Relation}

The relativistic equation to describe the motion of a charge particle under an external force $\boldsymbol{F}$ is given by [6]

$$
\frac{\mathrm{d} \gamma m \boldsymbol{v}}{\mathrm{d} t}=\boldsymbol{F},
$$

where $m$ and $\boldsymbol{v}$ are the mass and the velocity of the charged particle, and $\gamma$ is the usual relativistic time dilation factor

$$
\gamma=\left(1-\beta^{2}\right)^{-1 / 2}
$$

being $\beta$ the speed of the charged particle in units of the speed of light " $c$ ",

$$
\beta=\frac{v}{c}=\frac{1}{c} \sqrt{v_{x}^{2}+v_{y}^{2}+v_{z}^{2}} .
$$

Making the differentiation in (1), this equation can be written as

$$
m \gamma^{3}\left(\begin{array}{ccc}
1-\beta_{y}^{2}-\beta_{z}^{2} & \beta_{x} \beta_{y} & \beta_{x} \beta_{z} \\
\beta_{y} \beta_{x} & 1-\beta_{x}^{2}-\beta_{z}^{2} & \beta_{y} \beta_{z} \\
\beta_{z} \beta_{x} & \beta_{y} \beta_{y} & 1-\beta_{x}^{2}-\beta_{y}^{2}
\end{array}\right)\left(\begin{array}{c}
\dot{v}_{x} \\
\dot{v}_{y} \\
\dot{v}_{x}
\end{array}\right)=\boldsymbol{F},
$$

where $\beta_{i}=v_{i} / c \quad i=x, y, z$. Inverting this matrix and making some rearrangements, it follows that

$$
\dot{\boldsymbol{\beta}}=\frac{1}{m c \gamma} \aleph \boldsymbol{F},
$$

where $\aleph$ is the matrix

$$
\aleph=\left(\begin{array}{ccc}
1-\beta_{x}^{2} & -\beta_{x} \beta_{y} & -\beta_{x} \beta_{z} \\
-\beta_{y} \beta_{x} & 1-\beta_{y}^{2} & -\beta_{y} \beta_{z} \\
-\beta_{z} \beta_{x} & -\beta_{z} \beta_{y} & 1-\beta_{z}^{2}
\end{array}\right) .
$$

This is the expression with makes the relation between the normalized acceleration with the external force.

\section{Radiation Reaction Force}

It is well known that the power radiated per solid angle of an accelerated charged particle of charge " $q$ " is [7]

$$
\frac{\mathrm{d} P}{\mathrm{~d} \Omega}=\frac{q^{2}}{4 \pi c} \frac{|\hat{\boldsymbol{R}} \times[(\hat{\boldsymbol{R}}-\boldsymbol{\beta}) \times \dot{\boldsymbol{\beta}}]|^{2}}{(1-\hat{\boldsymbol{R}} \cdot \boldsymbol{\beta})^{5}},
$$

where $\hat{\boldsymbol{R}}$ is the unitary vector that goes from the position of the charge at the retarded time $\left(t^{\prime}=t+R / c\right)$ to the observer position. So, using (5) on this expression, it follows that 


$$
\frac{\mathrm{d} P}{\mathrm{~d} \Omega}=\frac{q^{2}}{4 \pi m^{2} c^{3} \gamma^{2}} \frac{|\hat{\boldsymbol{R}} \times[(\hat{\boldsymbol{R}}-\boldsymbol{\beta}) \times \mathfrak{\aleph} \boldsymbol{F}]|^{2}}{(1-\hat{\boldsymbol{R}} \cdot \boldsymbol{\beta})^{5}},
$$

Integrating with respect the solid angle and with respect the time in the interval $[0, t]$, the energy radiated by the charged particle during this time is

$$
U(t)=\frac{q^{2}}{4 \pi m^{2} c^{3}} \int_{0}^{t} \frac{\mathrm{d} t}{\gamma^{2}} \int_{\Omega} \frac{|\hat{\boldsymbol{R}} \times[(\hat{\boldsymbol{R}}-\boldsymbol{\beta}) \times \aleph \boldsymbol{F}]|^{2}}{(1-\hat{\boldsymbol{R}} \cdot \boldsymbol{\beta})^{5}} \mathrm{~d} \Omega .
$$

which can be written in terms of angles as

$$
U(t)=\frac{q^{2}}{4 \pi m^{2} c^{3}} \int_{0}^{t} \frac{|\boldsymbol{N} \boldsymbol{F}|^{2} \mathrm{~d} t}{\gamma^{2}} \int_{\Omega} \frac{\left|e_{1} \sin \theta_{1}-e_{2} \beta \sin \theta_{2}\right|^{2}}{(1-\beta \cos \theta)^{5}} \mathrm{~d} \Omega,
$$

where $e_{1}$ is the unitary vector in the direction $\hat{\boldsymbol{R}} \times(\hat{\boldsymbol{R}} \times \mathfrak{N} \boldsymbol{F})$ and $e_{2}$ is the unitary vector in the direction $\hat{\boldsymbol{R}} \times(\boldsymbol{\beta} \times \boldsymbol{N} \boldsymbol{F}), \theta_{1}$ is the angle between the vectors $\hat{\boldsymbol{R}}$ and $\hat{\boldsymbol{R}} \times \mathbf{N} \boldsymbol{F}$, and $\theta_{2}$ is the angle between the vectors $\hat{\boldsymbol{R}}$ and $\boldsymbol{\beta} \times \boldsymbol{\aleph} \boldsymbol{F}$. One can choose our reference system such that $\theta$ be the angle related with the solid angle coordinates. The angles $\theta_{1}$ and $\theta_{2}$ depend on the solid angle coordinates $(\theta, \phi)$.

Assume now that this energy lost is due to the work done by a nonconservative radiation reaction force, to move the charged particle from the position $x_{0}$ a the time $t=0$, to the position $\boldsymbol{x}$ at the time t. So, one would have

$$
U(t)=\int_{x_{0}}^{x} F_{r a d} \cdot \mathrm{d} \boldsymbol{x} .
$$

However, one has that $\mathrm{d} \boldsymbol{x}=\boldsymbol{v} \mathrm{d} t$. Then, it follows that

$$
U(t)=\int_{0}^{t} \boldsymbol{F}_{\text {rad }} \cdot \boldsymbol{v} \mathrm{d} t .
$$

Equaling (10) and (12), and since the resulting expression is valid for any time intervale $[0, t]$ on the real line, one obtains

$$
\boldsymbol{F}_{\text {rad }}=\frac{q^{2}|\boldsymbol{N} \boldsymbol{F}|^{2}}{4 \pi m^{2} c^{3} \gamma^{2} v \cos \varphi} \int_{\Omega} \frac{\left|e_{1} \sin \theta_{1}-e_{2} \beta \sin \theta_{2}\right|^{2}}{(1-\beta \cos \theta)^{5}} \mathrm{~d} \Omega,
$$

where $V$ is the charged particle speed and $\varphi$ is the angle between the vectors $\boldsymbol{F}_{\text {rad }}$ and $\boldsymbol{v}$. Since $\boldsymbol{F}_{\text {rad }}$ must represent a force causing damping on the motion of the charged particle, $\boldsymbol{F}_{\text {rad }}$ must point on the $-\hat{\boldsymbol{n}}$ direction, where $\hat{\boldsymbol{n}}=\boldsymbol{v} / v$, meaning the the angle between $\boldsymbol{F}_{\text {rad }}$ and $\boldsymbol{v}$ must be $\varphi=\pi$. Therefore, one gets the following expression for radiation reaction force

$$
\boldsymbol{F}_{\text {rad }}=-\frac{q^{2}|\boldsymbol{\kappa} \boldsymbol{F}|^{2} \boldsymbol{v}}{4 \pi m^{2} c^{3} \gamma^{2} v^{2}} \int_{\Omega} \frac{\left|e_{1} \sin \theta_{1}-e_{2} \beta \sin \theta_{2}\right|^{2}}{(1-\beta \cos \theta)^{5}} \mathrm{~d} \Omega,
$$

In this way, the modified relativistic equation of motion of a charged particle under an arbitrary external force $\boldsymbol{F}$ is

$$
\frac{\mathrm{d} \gamma m \boldsymbol{v}}{\mathrm{d} t}=\boldsymbol{F}+\boldsymbol{F}_{r a d},
$$

where the radiation reaction force term $\boldsymbol{F}_{\text {rad }}$ has been added to the expression 
(1), or it can be written, using (14), as

$$
\frac{\mathrm{d} \gamma m \boldsymbol{v}}{\mathrm{d} t}=\boldsymbol{F}-\frac{q^{2}|\boldsymbol{\aleph} \boldsymbol{F}|^{2} \boldsymbol{v}}{4 \pi m^{2} c^{3} \gamma^{2} v^{2}} \int_{\Omega} \frac{\left|e_{1} \sin \theta_{1}-e_{2} \beta \sin \theta_{2}\right|^{2}}{(1-\beta \cos \theta)^{5}} \mathrm{~d} \Omega .
$$

One must point out that if the external force $\boldsymbol{F}$ is zero, the radiation reaction force $\boldsymbol{F}_{\text {rad }}$ is also zero, and noncausal preacceleration is absent since the charged particle will have constant velocity, as the experiments indicate so far.

\section{Special Cases}

As one can see from expression (14), the integration over the solid angle is not in general a trivial matter, and numerical integration may be required. However, there are two cases where this integration can be done without any difficulty, and these cases are presented below.

a) Linear acceleration case: In this case one has that $\boldsymbol{N F}$ is parallel to $\boldsymbol{\beta}$ $\left(e_{2}=0\right)$ and there is not dependence on $\phi$, this integration is well known [7], and choosing $\boldsymbol{\beta}=\left(0,0, \beta_{z}\right)$ and $\boldsymbol{F}=(0,0, F)$, the resulting equation is

$$
\frac{\mathrm{d} \gamma m v}{\mathrm{~d} t}=F-\frac{\lambda_{0} F^{2}}{v}
$$

where $\gamma=\left(1-\beta_{z}^{2}\right)^{-1 / 2}$, and $\lambda_{0}$ is defined as

$$
\lambda_{0}=\frac{2 q^{2}}{3 m^{2} c^{3}} \text {. }
$$

This equation is the same as that proposed for this case in reference [5].

b) Circular acceleration case: In this case one has that $\boldsymbol{\aleph F}$ and $\boldsymbol{\beta}$ are orthogonal. The integration is also well known [7], and choosing $\boldsymbol{\beta}=\left(\beta_{x}, \beta_{y}, 0\right)$ and $\boldsymbol{F}=\left(F_{x}, F_{y}, 0\right)$, one arrives to the following equation

$$
\frac{\mathrm{d} \gamma m \boldsymbol{v}}{\mathrm{d} t}=\boldsymbol{F}-\frac{\lambda_{0} F^{2}}{v^{2} \gamma^{2}} \boldsymbol{v}
$$

where $\gamma=\left(1-\beta_{x}^{2}-\beta_{y}^{2}\right)^{-1 / 2}, v^{2}=v_{x}^{2}+v_{y}^{2}$, and the motion is reduced to the plane $(x, y)$. This expression is the same as that one proposed on reference [5].

\section{Conclusion}

Following the same approach of reference [5], a generalization has been formulated for the force approach to radiation reaction, expression (16). This generalization has the same main property of the force approach, wherever the external force is zero, the acceleration of the charged particle is zero, and the radiation reaction force is zero too, and non pre-acceleration concept will appear here.

\section{References}

[1] Jackson, J.D. (2007) Comment on "Preacceleration without radiation: The nonexistence of preradiation phenomenon," by J. A. Heras [Am. J. Phys. 74 (11), 1025-1030 (2006)]. American Journal of Physics, 75, 844. https://doi.org/10.1119/1.2733681

[2] Hnizdo, V. (2007) Comment on "Preacceleration without radiation: The nonexistence of preradiation phenomenon," by J. A. Heras [Am. J. Phys. 74 (11), 1025-1030 (2006)]. American Journal of Physics, 75, 845. https://doi.org/10.1119/1.2733682 
[3] Heras, J.A. (2007) Can Maxwell's Equations Be Obtained from the Continuity Equation? American Journal of Physics, 75, 652. https://doi.org/10.1119/1.2739570

[4] Griffiths, D.J., Proctor, T.C. and Shroeter, D.F. (2010) Abraham-Lorentz versus Landau-Lifshitz. American Journal of Physics, 78, 391. https://doi.org/10.1119/1.3269900

[5] Gustavo, V. (2016) López, Force Approach to Radiation Reaction. Annals of Physics, 365, 1-6. https://doi.org/10.1016/j.aop.2015.11.012

[6] Møller, C. (1952) The Theory of Relativity. Oxford University Press, Oxford.

[7] Jackson, J.D. (1962) Classical Electrodynamics. John Wiley \& Sons Inc.

Submit or recommend next manuscript to SCIRP and we will provide best service for you:

Accepting pre-submission inquiries through Email, Facebook, LinkedIn, Twitter, etc. A wide selection of journals (inclusive of 9 subjects, more than 200 journals)

Providing 24-hour high-quality service

User-friendly online submission system

Fair and swift peer-review system

Efficient typesetting and proofreading procedure

Display of the result of downloads and visits, as well as the number of cited articles Maximum dissemination of your research work

Submit your manuscript at: http://papersubmission.scirp.org/

Or contact jamp@scirp.org 